\title{
The Effect of Magnetic Field and Initial Stress on Fractional Order Generalized Thermoelastic Half-Space
}

\author{
Sunita Deswal, Sandeep Singh Sheoran, and Kapil Kumar Kalkal \\ Department of Mathematics, G. J. University of Science and Technology, Haryana, Hisar 125001, India \\ Correspondence should be addressed to Kapil Kumar Kalkal; kapilkalkal_gju@rediffmail.com
}

Received 26 December 2012; Revised 5 March 2013; Accepted 12 March 2013

Academic Editor: Petr Ekel

Copyright (C) 2013 Sunita Deswal et al. This is an open access article distributed under the Creative Commons Attribution License, which permits unrestricted use, distribution, and reproduction in any medium, provided the original work is properly cited.

\begin{abstract}
The aim of this paper is to study magneto-thermoelastic interactions in an initially stressed isotropic homogeneous half-space in the context of fractional order theory of generalized thermoelasticity. State space formulation with the Laplace transform technique is used to obtain the general solution, and the resulting formulation is applied to the ramp type increase in thermal load and zero stress. Solutions of the problem in the physical domain are obtained by using a numerical method of the Laplace inverse transform based on the Fourier expansion technique, and the expressions for the displacement, temperature, and stress inside the half-space are obtained. Numerical computations are carried out for a particular material for illustrating the results. Results obtained for the field variables are displayed graphically. Some comparisons have been shown in figures to present the effect of fractional parameter, ramp parameter, magnetic field, and initial stress on the field variables. Some particular cases of special interest have been deduced from the present investigation.
\end{abstract}

\section{Introduction}

Biot [1] developed the coupled theory of thermoelasticity to overcome the paradox inherent in the uncoupled theory that elastic changes have no effect on temperature. In this theory, the equations of elasticity and heat conduction are coupled. But, it shares the defect of the uncoupled theory in which it predicts infinite speed of propagation for heat waves. Generalized thermoelastic theories have been developed with the objective of removing the defect of coupled theory. At present, mainly two different models of generalized thermoelasticity are being extensively used, one proposed by Lord and Shulman [2] and the other proposed by Green and Lindsay [3]. The Lord-Shulman theory only modified the Fourier heat conduction equation and suggested one relaxation time, whereas the Green-Lindsay theory modified both the energy equation and the equation of motion and suggested two relaxation times. Dhaliwal and Sherief [4] extended the LordShulman theory to include anisotropic case. Hetnarski and Ignaczak [5] presented a survey article of various representative theories in the range of generalized thermoelasticity. Youssef [6] studied two-dimensional generalized thermoelasticity problem for half-space subjected to ramp type heating.
The theory of magneto-thermoelasticity has received attention of many researchers due to its application in widely diverse fields such as geophysics for understanding the effect of earth's magnetic field on seismic waves, damping of acoustic waves, emission of electromagnetic radiations from nuclear devices, optics, and so forth. The theory of magnetothermoelasticity was introduced by Knopoff [7] and Chadwick [8] and developed by Kaliski and Petykiewicz [9]. The theoretical outline of the development of magneto-thermoelasticity was discussed by Paria [10]. Paria studied the propagation of plane magneto-thermoelastic waves in an isotropic unbounded medium under the influence of a magnetic field acting transversely to the direction of propagation. Nayfeh and Nemat-Nasser [11] studied the propagation of plane waves in a solid under the influence of electro-magnetic field. Sherief and Ezzat [12] discussed a thermal shock problem in magneto-thermoelasticity with thermal relaxation. Sherief and Helmy [13] illustrated a two-dimensional half-space problem in the context of electromagneto-thermoelasticity theory subjected to a nonuniform thermal shock. Ezzat and Youssef [14] constructed a problem on generalized magneto-thermoelasticity in a perfectly conducting medium. Baksi et al. [15] examined magneto-thermoelastic 
problem with thermal relaxation and heat source in threedimensional, infinite rotating elastic medium.

The development of initial stress in the medium is due to many reasons such as the process of quenching, resulting from difference of temperatures, slow process of creep, differential external forces, and gravity variations. The earth is supposed to be under high initial stress. The researchers have shown much interest to study the effect of these stresses on the propagation of waves. Biot [16] solved the dynamic problem of elastic medium under initial stress. Chattopadhyay et al. [17] studied the reflection of elastic waves under initial stress at a free surface. Montanaro [18] studied the isotropic linear thermoelasticity with hydrostatic initial stress by using Biot's linearization of the constitutive law for stress. Othman and Song [19] investigated the reflection of plane waves from an elastic solid half-space under hydrostatic initial stress without energy dissipation. Singh [20] explored the effect of hydrostatic initial stress on waves in a thermoelastic half-space.

The theory of fractional derivative and integral was established in the second half of nineteenth century. The first application of fractional derivative was given by Abel who applied fractional calculus in the solution of an integral equation that arises in the formulation of tautochrone problem. In the recent years, fractional calculus has been applied successfully in various areas to modify many existing models of physical processes such as heat conduction, diffusion, viscoelasticity, wave propagation, and electronics. Caputo and Mainardi [21, 22] and Caputo [23] have established the relation between fractional derivative and theory of linear viscoelasticity. The generalization of the concept of derivative and integral to a noninteger order has been subjected to several approaches, and some various alternative definitions of fractional derivatives appeared in [24-27]. One can refer to Podlubny [28] for a survey of applications of fractional calculus. Povstenko [29] has proposed a quasistatic uncoupled theory of thermoelasticity based on fractional heat conduction equation. Sherief et al. [30] introduced a new model of thermoelasticity using fractional calculus, proved a uniqueness theorem, and derived a reciprocity relation and a variational principle. In this model, heat conduction equation takes the form as

$$
q_{i}+\tau_{0} \frac{\partial^{\alpha}}{\partial t^{\alpha}} q_{i}=-k_{i j} \theta_{, j}
$$

where $q_{i}$ are the components of the heat flux vector, $\theta$ is the temperature, $\tau_{0}$ is the thermal relaxation time parameter, $k_{i j}$ is thermal conductivity tensor, and $\alpha$ is a fractional parameter such that $0<\alpha \leq 1$. The above heat conduction equation reduces to the Maxwell-Cattaneo law in the limiting case when $\alpha \rightarrow 1$. It should be mentioned here that the MaxwellCattaneo law has been employed by Lord and Shulman [2] to develop first generalized theory of thermoelasticity. Youssef [31] constructed another model of thermoelasticity in the context of a new consideration of heat conduction with a fractional order and proved the uniqueness theorem. In this model, Youssef described different cases of conductivity: $0<$ $\alpha<1$ corresponds to weak conductivity, $\alpha=1$ corresponds to normal conductivity, and $1<\alpha \leq 2$ corresponds to superconductivity. Ezzat $[32,33]$ established a model of fractional heat conduction equation by using the new Taylor series expansion of time-fractional order developed by Jumarie [34]. ElKaramany and Ezzat [35] introduced two general models of fractional heat conduction law for a nonhomogeneous anisotropic elastic solid. Uniqueness and reciprocal theorems are proved, and the convolutional variational principle is established and used to prove a uniqueness theorem with no restriction on the elasticity or thermal conductivity tensors except symmetry conditions. The two-temperature dynamic coupled, Lord-Shulman and fractional coupled thermoelasticity theories, result as limit cases. For fractional thermoelasticity not involving two-temperatures, El-Karamany and Ezzat [36] established the uniqueness, reciprocal theorems and convolution variational principle. The dynamic coupled and the Green-Naghdi thermoelasticity theories result as limit cases. The reciprocity relation in case of quiescent initial state is found to be independent of the order of differintegration $[35,36]$. Fractional order theory of a perfect conducting thermoelastic medium not involving two temperatures was investigated by El-Karamany and Ezzat [37]. Kothari and Mukhopadhyay [38] studied a half-space problem under fractional order theory of thermoelasticity and analyzed the effect of the fractional order parameter on the field variables.

In the present paper, we study the effect of magnetic field and initial stress under fractional order theory of thermoelasticity proposed by Sherief et al. [30]. We employ a state space approach developed by Bahar and Hetnarski [39] on the formulation. The Laplace transform technique is used to obtain the general solution. The inverse Laplace transform is carried out using a numerical inversion method developed by Honig and Hirdes [40]. Finally, the effect of fractional parameter, ramp parameter, magnetic field, and initial stress on field variables is displayed graphically.

\section{Governing Equations}

The governing equations in the context of fractional order theory of generalized thermoelasticity with initial stress and magnetic field for isotropic and homogeneous elastic medium are considered as

(i) the equation of motion

$$
\rho \ddot{u}_{i}=\sigma_{j i, j}+F_{i},
$$

where $\vec{F}=\mu_{0} \vec{J} \times \vec{H}$,

(ii) heat conduction equation

$k \theta_{i i}=\rho c_{E}\left(1+\tau_{0} \frac{\partial^{\alpha}}{\partial t^{\alpha}}\right) \frac{\partial \theta}{\partial t}+\beta T_{0}\left(1+\tau_{0} \frac{\partial^{\alpha}}{\partial t^{\alpha}}\right) \frac{\partial u_{i, i}}{\partial t}$,

(iii) constitutive relations

$$
\begin{gathered}
\sigma_{i j}=-p\left(\delta_{i j}+\omega_{i j}\right)+2 \bar{\mu} e_{i j}+\bar{\lambda} e \delta_{i j}-\beta \theta \delta_{i j}, \\
e_{i j}=\frac{1}{2}\left(u_{i, j}+u_{j, i}\right), \\
\omega_{i j}=\frac{1}{2}\left(u_{j, i}-u_{i, j}\right) .
\end{gathered}
$$


We take the linearized Maxwell equations governing the electromagnetic field for a perfectly conducting medium as

$$
\begin{gathered}
\operatorname{curl} \vec{h}=\vec{J}+\varepsilon_{0} \frac{\partial \vec{E}}{\partial t}, \\
\operatorname{curl} \vec{E}=-\mu_{0} \frac{\partial \vec{h}}{\partial t}, \\
\vec{E}=-\mu_{0}\left(\frac{\partial \vec{h}}{\partial t} \times \vec{H}\right), \\
\operatorname{div} \vec{h}=0,
\end{gathered}
$$

where $u_{i}$ are the components of displacement vector $\vec{u}, \theta=$ $T-T_{0}, T$ is the absolute temperature, $T_{0}$ is the reference temperature assumed to obey the inequality $\left|\theta / T_{0}\right| \ll 1, \tau_{0}$ is the thermal relaxation time, $\sigma_{i j}$ are the components of the stress tensor, $e_{i j}$ are the components of strain tensor, $\delta_{i j}$ is the Kronecker delta function, $e$ is the cubical dilation, $\rho$ is the density of the medium, $c_{E}$ is the specific heat, $k$ is the thermal conductivity, $\beta=(3 \bar{\lambda}+2 \bar{\mu}) \alpha_{t}$, $\alpha_{t}$ is the coefficient of linear thermal expansion, $\bar{\lambda}$ and $\bar{\mu}$ are the lame constants, $p$ is the initial stress, $F_{i}$ are the components of Lorentz's body force vector $\vec{F}, \mu_{0}$ is the magnetic permeability, $\varepsilon_{0}$ is the electric permittivity, $\vec{H}$ is the applied magnetic field, $\vec{h}$ is the induced magnetic field, $\vec{E}$ is the induced electric field, and $\vec{J}$ is the current density vector.

\section{Problem Formulation}

We consider a perfectly conducting isotropic homogeneous and fractional order generalized thermoelastic half-space with hydrostatic initial stress subjected to a constant magnetic field $\vec{H}\left(0, H_{0}, 0\right)$ which produces an induced magnetic field $\vec{h}\left(0, h_{2}, 0\right)$ and induced electric field $\vec{E}\left(0,0, E_{3}\right)$. We assume one-dimensional motion for which all the field quantities are functions of $x$ and $t$.

The displacement components take the form

$$
u_{x}=u(x, t), \quad u_{y}=u_{z}=0 .
$$

The strain component becomes

$$
e=e_{x x}=\frac{\partial u}{\partial x} .
$$

The components of magnetic field vectors are

$$
H_{x}=0, \quad H_{y}=H_{0}, \quad H_{z}=0 .
$$

The electric intensity vector $\vec{E}$ is parallel to current density vector $\vec{J}$. Hence, components of $\vec{E}$ and $\vec{J}$ are given as

$$
\begin{array}{cc}
E_{x}=E_{y}=0, & E_{z}=E_{3}, \\
J_{x}=J_{y}=0, & J_{z}=J_{3} .
\end{array}
$$

Now, the Maxwell equation (5) provides the following results:

$$
E_{3}=-\mu_{0} H_{0} \frac{\partial u}{\partial t}, \quad h_{2}=-H_{0} e, \quad J_{3}=\varepsilon_{0} \mu_{0} H_{0} \frac{\partial^{2} u}{\partial^{2} t} .
$$

Using (8) and (10) into the relation $\vec{F}=\mu_{0} \vec{J} \times \vec{H}$, we obtain

$$
F_{x}=-\varepsilon_{0} \mu_{0}^{2} H_{0}^{2} \ddot{u}, \quad F_{y}=F_{z}=0 .
$$
come

The governing equations for one-dimensional case be-

$$
\begin{gathered}
\rho \ddot{u}=\frac{\partial \sigma}{\partial x}+F_{x}, \\
k \frac{\partial^{2} \theta}{\partial x^{2}}=\rho c_{E}\left(1+\tau_{0} \frac{\partial^{\alpha}}{\partial t^{\alpha}}\right) \frac{\partial \theta}{\partial t}+\beta T_{0}\left(1+\tau_{0} \frac{\partial^{\alpha}}{\partial t^{\alpha}}\right) \frac{\partial e}{\partial t}, \\
\sigma=\sigma_{x x}=-p+(\bar{\lambda}+2 \bar{\mu}) e-\beta \theta .
\end{gathered}
$$

Now, we will use the following nondimensional variables:

$$
\begin{gathered}
x^{\prime}=c_{0} \eta_{0} x, \quad u^{\prime}=c_{0} \eta_{0} u, \\
t^{\prime}=c_{0}^{2} \eta_{0} t, \quad \tau_{0}^{\prime}=c_{0}^{2} \eta_{0} \tau_{0}, \\
\theta^{\prime}=\frac{\theta}{T_{0}}, \quad \sigma^{\prime}=\frac{\sigma}{\bar{\lambda}+2 \mu}, \quad p^{\prime}=\frac{p}{\beta T_{0}},
\end{gathered}
$$

where

$$
\eta_{0}=\frac{\rho c_{E}}{k}, \quad c_{0}^{2}=\frac{\bar{\lambda}+2 \bar{\mu}}{\rho} .
$$

Expressing (12)-(14) in terms of the nondimensional variables given by (15) and dropping the prime sign for convenience, we have the following forms:

$$
\begin{gathered}
\frac{\partial^{2} \sigma}{\partial x^{2}}=\alpha_{1} \ddot{e}, \\
\frac{\partial^{2} \theta}{\partial x^{2}}=\left(1+\tau_{0}^{*} \frac{\partial^{\alpha}}{\partial t^{\alpha}}\right)\left(\dot{\theta}+\beta_{1} \dot{e}\right), \\
\sigma=e-\varepsilon_{1} \theta-\delta_{1},
\end{gathered}
$$

where

$$
\begin{gathered}
\alpha_{1}=1+\frac{\varepsilon_{0} \mu_{0}^{2} H_{0}^{2}}{\rho}, \quad \beta_{1}=\frac{\beta}{\rho c_{E}}, \quad \varepsilon_{1}=\frac{\beta T_{0}}{\bar{\lambda}+2 \bar{\mu}}, \\
\delta_{1}=\varepsilon_{1} p, \quad \tau_{0}^{*}=\frac{\tau_{0}}{\left(c_{0}^{2} \eta_{0}\right)^{1-\alpha}} .
\end{gathered}
$$

These equations will be supplemented with appropriate boundary conditions relevant to the particular application under consideration.

Taking the Laplace transform of (17) by using homogeneous initial conditions defined and denoted as

$$
\bar{f}(s)=\int_{0}^{\infty} e^{-s t} f(t) d t, \quad s>0,
$$


we obtain

$$
\begin{gathered}
\frac{\partial^{2} \bar{\sigma}}{\partial x^{2}}=\alpha_{1} s^{2} \bar{e}, \\
\frac{\partial^{2} \bar{\theta}}{\partial x^{2}}=\left(s+\tau_{0}^{*} s^{\alpha+1}\right)\left(\bar{\theta}+\beta_{1} \bar{e}\right), \\
\bar{\sigma}=\bar{e}-\varepsilon_{1} \bar{\theta}-\frac{\delta_{1}}{s} .
\end{gathered}
$$

Eliminating the value of $\bar{e}$ from (20) and (21) by using (22), we obtain

$$
\begin{gathered}
\frac{\partial^{2} \bar{\theta}}{\partial x^{2}}=L_{1} \bar{\theta}+L_{2} \bar{\sigma}+L_{3}, \\
\frac{\partial^{2} \bar{\sigma}}{\partial x^{2}}=M_{1} \bar{\theta}+M_{2} \bar{\sigma}+M_{3},
\end{gathered}
$$

where

$$
\begin{gathered}
L_{1}=\left(1+\beta_{1} \varepsilon_{1}\right)\left(s+\tau_{0}^{*} s^{\alpha+1}\right), \quad L_{2}=\beta_{1}\left(s+\tau_{0}^{*} s^{\alpha+1}\right), \\
L_{3}=\beta_{1} \delta_{1}\left(1+\tau_{0}^{*} s^{\alpha}\right), \\
M_{1}=\alpha_{1} \varepsilon_{1} s^{2}, \quad M_{2}=\alpha_{1} s^{2}, \quad M_{3}=\alpha_{1} \delta_{1} s .
\end{gathered}
$$

\section{State-Space Formulation}

Now, choosing the temperature of heat conduction $\theta$ and the stress component $\sigma$ in $x$-direction as state variables, one can write (23) in matrix form as

$$
\frac{d^{2}}{d x^{2}} \bar{V}(x, s)=A(s) \bar{V}(x, s)+C(x, s),
$$

where

$$
\begin{gathered}
A(s)=\left[\begin{array}{cc}
L_{1} & L_{2} \\
M_{1} & M_{2}
\end{array}\right], \quad \bar{V}(x, s)=\left[\begin{array}{l}
\bar{\theta}(x, s) \\
\bar{\sigma}(x, s)
\end{array}\right], \\
C(x, s)=\left[\begin{array}{c}
L_{3} \\
M_{3}
\end{array}\right] .
\end{gathered}
$$

The formal solution of (25) can be written in the form

$$
\begin{aligned}
\bar{V}(x, s)= & \exp [-\sqrt{A(s)} x]\left[\bar{V}(0, s)+C(0, s) A^{-1}(s)\right] \\
& -C(x, s) A^{-1}(s),
\end{aligned}
$$

where

$$
\bar{V}(0, s)=\left[\begin{array}{l}
\bar{\theta}(0, s) \\
\bar{\sigma}(0, s)
\end{array}\right]=\left[\begin{array}{l}
\bar{\theta}_{0} \\
\bar{\sigma}_{0}
\end{array}\right] .
$$

In the above solution, we have cancelled the part of exponential having positive power to get bounded solution for large $x$.

Now, we will use the Cayley-Hamilton theorem to find the form of the matrix $\exp [-\sqrt{A(s)} x]$, and, for this, we proceed as follows.
The characteristic equation of the matrix $A(s)$ is obtained as

$$
\lambda^{2}-\left(L_{1}+M_{2}\right) \lambda+\left(L_{1} M_{2}-M_{1} L_{2}\right)=0,
$$

where the roots of (29), namely, $\lambda_{1}$, and $\lambda_{2}$ satisfy the following relations:

$$
\begin{gathered}
\lambda_{1}+\lambda_{2}=L_{1}+M_{2}, \\
\lambda_{1} \lambda_{2}=L_{1} M_{2}-L_{2} M_{1} .
\end{gathered}
$$

Now, we write the spectral decomposition of matrix $A(s)$ as

$$
A(s)=\lambda_{1} E+\lambda_{2} F,
$$

where $E$ and $F$ are called the projectors of $A(s)$ and satisfy the following conditions

$$
\begin{gathered}
E+F=I, \\
E F=F E=O, \\
E^{2}=E, \quad F^{2}=F .
\end{gathered}
$$

Then, we have

$$
\sqrt{A(s)}=\sqrt{\lambda_{1}} E+\sqrt{\lambda_{2}} F
$$

where

$$
\begin{gathered}
E=-\frac{1}{\lambda_{1}-\lambda_{2}}\left[\begin{array}{cc}
\lambda_{2}-L_{1} & -L_{2} \\
-M_{1} & \lambda_{2}-M_{2}
\end{array}\right], \\
F=\frac{1}{\lambda_{1}-\lambda_{2}}\left[\begin{array}{cc}
\lambda_{1}-L_{1} & -L_{2} \\
-M_{1} & \lambda_{1}-M_{2}
\end{array}\right], \\
I=\left[\begin{array}{ll}
1 & 0 \\
0 & 1
\end{array}\right], \quad O=\left[\begin{array}{ll}
0 & 0 \\
0 & 0
\end{array}\right] .
\end{gathered}
$$

Finally, we get

$$
B(s)=\sqrt{A(s)}=\frac{1}{\sqrt{\lambda_{1}}+\sqrt{\lambda_{2}}}\left[\begin{array}{cc}
\sqrt{\lambda_{1} \lambda_{2}}+L_{1} & L_{2} \\
M_{1} & \sqrt{\lambda_{1} \lambda_{2}}+M_{2}
\end{array}\right] .
$$

The characteristic equation of matrix $B(s)$ can be written as

$$
k^{2}-k\left(\sqrt{\lambda_{1}}+\sqrt{\lambda_{2}}\right)+\sqrt{\lambda_{1}} \sqrt{\lambda_{2}}=0,
$$

where the roots of (36), namely, $k_{1}$ and $k_{2}$, can be written as

$$
k_{1}=\sqrt{\lambda_{1}}, \quad k_{2}=\sqrt{\lambda_{2}} .
$$

Now, the Taylor series expansion of $\exp [-\sqrt{A(s)} x]$ yields

$$
\exp [-\sqrt{A(s)} x]=\exp [-B(s) x]=\sum_{n=0}^{\infty} \frac{[-B(s) x]^{n}}{n !} .
$$


Using the Cayley-Hamilton theorem, we can express $B^{2}$ and higher orders of matrix $B$ in terms of $I$ and $B$, where $I$ is the identity matrix of second order, Bahar and Hetnarski [39].

Therefore, the infinite series in (38) can be expressed as

$$
L(x, s)=\exp [-B(s) x]=b_{0} I+b_{1} B(s),
$$

where $b_{0}$ and $b_{1}$ are coefficients depending on $x$ and $s$. By the Cayley-Hamilton theorem, the characteristic roots $k_{1}$ and $k_{2}$ of the matrix $B$ must satisfy (39), so we have

$$
\begin{aligned}
& \exp \left[-k_{1} x\right]=b_{0}+b_{1} k_{1}, \\
& \exp \left[-k_{2} x\right]=b_{0}+b_{1} k_{2} .
\end{aligned}
$$

By solving the above system of equations and using (37), we get

$$
\begin{gathered}
b_{0}=\frac{\sqrt{\lambda_{1}} e^{-\sqrt{\lambda_{2}} x}-\sqrt{\lambda_{2}} e^{-\sqrt{\lambda_{1}} x}}{\sqrt{\lambda_{1}}-\sqrt{\lambda_{2}}}, \\
b_{1}=\frac{e^{-\sqrt{\lambda_{1}} x}-e^{-\sqrt{\lambda_{2}} x}}{\sqrt{\lambda_{1}}-\sqrt{\lambda_{2}}} .
\end{gathered}
$$

Plugging the values of $b_{0}$ and $b_{1}$ in (39), we have

$$
\exp [-B(s) x]=L(x, s)=\left[l_{i j}\right], \quad i, j=1,2,
$$

where the entries $l_{i j}(x, s)$ are given as

$$
\begin{gathered}
l_{11}=\frac{\left(\lambda_{1}-L_{1}\right) e^{-\sqrt{\lambda_{2}} x}-\left(\lambda_{2}-L_{1}\right) e^{-\sqrt{\lambda_{1}} x}}{\lambda_{1}-\lambda_{2}}, \\
l_{12}=L_{2}\left(\frac{e^{-\sqrt{\lambda_{1}} x}-e^{-\sqrt{\lambda_{2}} x}}{\lambda_{1}-\lambda_{2}}\right), \\
l_{21}=M_{1}\left(\frac{e^{-\sqrt{\lambda_{1}} x}-e^{-\sqrt{\lambda_{2}} x}}{\lambda_{1}-\lambda_{2}}\right), \\
l_{22}=\frac{\left(\lambda_{1}-M_{2}\right) e^{-\sqrt{\lambda_{2}} x}-\left(\lambda_{2}-M_{2}\right) e^{-\sqrt{\lambda_{1}} x}}{\lambda_{1}-\lambda_{2}} .
\end{gathered}
$$

The solution of (25) can be written in the following form:

$$
\bar{V}(x, s)=\left[l_{i j}\right]\left[\bar{V}(0, s)+A^{-1}(s) C(0, s)\right]-A^{-1}(s) C(x, s) .
$$

Substituting the values of $\bar{V}(x, s), A^{-1}(s)$, and $C(x, s)$ into (44) and performing the necessary matrix operations, we obtain the values of $\bar{\theta}(x, s)$ and $\bar{\sigma}(x, s)$ as

$$
\begin{aligned}
\bar{\theta}(x, s)= & \frac{1}{\lambda_{1}-\lambda_{2}} \\
& \times\left[\left\{\left(\lambda_{1}-L_{1}\right)\left(\bar{\theta}_{0}+\eta_{1}\right)-L_{2}\left(\bar{\sigma}_{0}+\eta_{2}\right)\right\} e^{-\sqrt{\lambda_{2}} x}\right. \\
& \left.\quad-\left\{\left(\lambda_{2}-L_{1}\right)\left(\bar{\theta}_{0}+\eta_{1}\right)-L_{2}\left(\bar{\sigma}_{0}+\eta_{2}\right)\right\} e^{-\sqrt{\lambda_{1}} x}\right] \\
& -\eta_{1}, \\
\bar{\sigma}(x, s)= & \frac{1}{\lambda_{1}-} \\
& \times\left[\left(\lambda_{2}-M_{2}\right)\left(\bar{\sigma}_{0}+\eta_{2}\right)-M_{1}\left(\bar{\theta}_{0}+\eta_{1}\right)\right\} e^{-\sqrt{\lambda_{2}} x} \\
& \quad-\left\{\left(\lambda_{2}-M_{2}\right)\left(\bar{\sigma}_{0}+\eta_{2}\right)-M_{1}\left(\bar{\theta}_{0}+\eta_{1}\right)\right\} \\
& \left.\times e^{-\sqrt{\lambda_{1}} x}\right]-\eta_{2},
\end{aligned}
$$

where

$$
\eta_{1}=\frac{M_{2} L_{3}-L_{2} M_{3}}{L_{1} M_{2}-M_{1} L_{2}}, \quad \eta_{2}=\frac{L_{1} M_{3}-M_{1} L_{3}}{L_{1} M_{2}-M_{1} L_{2}} .
$$

Now, considering (12) along with (15), and the Laplace transform, the displacement component is evaluated as

$$
\bar{u}(x, s)=\frac{1}{\alpha_{1} s^{2}} \frac{\partial \bar{\sigma}}{\partial x} .
$$

Substituting (46) into (48), we get

$$
\begin{aligned}
& \bar{u}(x, s) \\
& =\frac{1}{\alpha_{1} s^{2}\left(\lambda_{1}-\lambda_{2}\right)} \\
& \times\left[\sqrt{\lambda_{1}}\left\{\left(\lambda_{2}-M_{2}\right)\left(\bar{\sigma}_{0}+\eta_{2}\right)-M_{1}\left(\bar{\theta}_{0}+\eta_{1}\right)\right\} e^{-\sqrt{\lambda_{1}} x}\right. \\
& \left.\quad-\sqrt{\lambda_{2}}\left\{\left(\lambda_{1}-M_{2}\right)\left(\bar{\sigma}_{0}+\eta_{2}\right)-M_{1}\left(\bar{\theta}_{0}+\eta_{1}\right)\right\} e^{-\sqrt{\lambda_{2}} x}\right] .
\end{aligned}
$$

\section{Application}

Problem: the ramp type boundary temperature of an elastic half-space.

We consider a homogeneous isotropic thermoelastic solid occupying the half-space $x \geq 0$. The boundary of half-space $x=0$ is affected by ramp type heating. In mathematical notations, the boundary conditions can be denoted as

$$
\begin{gathered}
\sigma(0, t)=\sigma_{0}=-p, \\
\theta(0, t)=\theta_{0}=\theta^{*} h(t),
\end{gathered}
$$


where $\theta^{*}$ is constant temperature and $h(t)$ is defined as

$$
h(t)= \begin{cases}0, & t \leq 0 \\ \frac{t}{t_{0},} & 0<t \leq t_{0} \\ 1, & t>t_{0}\end{cases}
$$

where $t_{0}$ is ramping parameter.

We employ nondimensional variables given in (15) on (50) and taking the Laplace transform, we get

$$
\begin{gathered}
\bar{\sigma}(0, s)=\bar{\sigma}_{0}=-\frac{\delta_{1}}{s}, \\
\bar{\theta}(0, s)=\bar{\theta}_{0}=\theta^{*}\left(\frac{1-e^{s t_{0}}}{t_{0} s^{2}}\right) .
\end{gathered}
$$

Substituting the values of $\bar{\sigma}_{0}$ and $\bar{\theta}_{0}$ from (52) into (45)(46) and (49), we find

$$
\begin{aligned}
& \bar{\theta}(x, s)=\frac{1}{\lambda_{1}-\lambda_{2}} \\
& \times\left[\left\{\left(\lambda_{1}-L_{1}\right)\left(\theta^{*} \eta^{*}+\eta_{1}\right)-L_{2}\left(\eta_{2}-\frac{\delta_{1}}{s}\right)\right\}\right. \\
& \times e^{-\sqrt{\lambda_{2}} x}-\left\{\left(\lambda_{2}-L_{1}\right)\left(\theta^{*} \eta^{*}+\eta_{1}\right)\right. \\
& \left.\left.-L_{2}\left(\eta_{2}-\frac{\delta_{1}}{s}\right)\right\} e^{-\sqrt{\lambda_{1}} x}\right]-\eta_{1} \\
& \bar{\sigma}(x, s)=\frac{1}{\lambda_{1}-\lambda_{2}} \\
& \times\left[\left\{\left(\lambda_{1}-M_{2}\right)\left(\eta_{2}-\frac{\delta_{1}}{s}\right)-M_{1}\left(\theta^{*} \eta^{*}+\eta_{1}\right)\right\}\right. \\
& \times e^{-\sqrt{\lambda_{2}} x}-\left\{\left(\lambda_{2}-M_{2}\right)\left(\eta_{2}-\frac{\delta_{1}}{s}\right)\right. \\
& \left.\left.-M_{1}\left(\theta^{*} \eta^{*}+\eta_{1}\right)\right\} e^{-\sqrt{\lambda_{1}} x}\right] \\
& -\eta_{2}, \\
& \bar{u}(x, s)=\frac{1}{\alpha_{1} s^{2}\left(\lambda_{1}-\lambda_{2}\right)} \\
& \times\left[\sqrt { \lambda _ { 1 } } \left\{\left(\lambda_{2}-M_{2}\right)\left(\eta_{2}-\frac{\delta_{1}}{s}\right)\right.\right. \\
& \left.-M_{1}\left(\theta^{*} \eta^{*}+\eta_{1}\right)\right\} e^{-\sqrt{\lambda_{1}} x} \\
& -\sqrt{\lambda_{2}}\left\{\left(\lambda_{1}-M_{2}\right)\left(\eta_{2}-\frac{\delta_{1}}{s}\right)\right. \\
& \left.\left.-M_{1}\left(\theta^{*} \eta^{*}+\eta_{1}\right)\right\} e^{-\sqrt{\lambda_{2}} x}\right],
\end{aligned}
$$

where

$$
\eta^{*}=\frac{1-e^{-s t_{0}}}{t_{0} s^{2}} .
$$

\section{Limiting Cases}

(1) The mathematical expressions for the field variables studied in the context of generalized magneto-thermoelasticity theory with initial stress under applied boundary condition can be obtained by applying $\alpha=$ 1 in (13).

(2) Neglecting initial stress effect, by substituting $p=0$ in (14) and $\eta=1$ in (58), we obtain the expressions of field variables under fractional order generalized magneto-thermoelasticity.

(3) The expressions for studied fields in the context of the fractional order generalized thermoelasticity theory with initial stress can be deduced by setting $H_{0}=0$ in (12).

\section{Numerical Inversion of the Laplace Transforms}

We will now outline the numerical inversion method used to find the solution in the physical domain. The inversion formula of the Laplace transform is defined as

$$
f(t)=\frac{1}{2 \pi \iota} \int_{c-\iota \infty}^{c+\iota \infty} e^{s t} \bar{f}(s) d s
$$

where $\bar{f}(s)$ is the Laplace transform of function $f(t)$.

In order to invert the Laplace transforms in the equations given in Section 5, we apply a numerical inversion method based on the Fourier series expansion explained by Honig and Hirdes [40]. In this method, the inverse transform $f(t)$ of the Laplace transform $\bar{f}(s)$ is approximated by the relation as

$$
\begin{array}{r}
f(t)=\frac{e^{c t}}{t_{1}}\left[\frac{1}{2} \bar{f}(c)+\operatorname{Re} \sum_{k=1}^{N} e^{\imath k \pi t / t_{1}} \bar{f}\left(c+\frac{\iota k \pi}{t_{1}}\right)\right], \\
0 \leq t<2 t_{1},
\end{array}
$$

where $N$ is a sufficiently large integer representing the number of terms in the truncated Fourier series chosen such that

$$
e^{c t} \operatorname{Re}\left[e^{\iota N \pi t / t_{1}} \bar{f}\left(c+\frac{\iota N \pi}{t_{1}}\right)\right] \leq \varepsilon_{1},
$$

where $\varepsilon_{1}$ is a prescribed small positive value that corresponds to the degree of accuracy to be achieved and $c$ is a positive constant and must be greater than the real parts of all the singularities of $\bar{f}(s)$. The optimal choice of $c$ was obtained according to the criteria described by Honig and Hirdes [40].

\section{Numerical Results and Discussions}

To illustrate and compare the theoretical results obtained in the Section 5, we now present some numerical results 


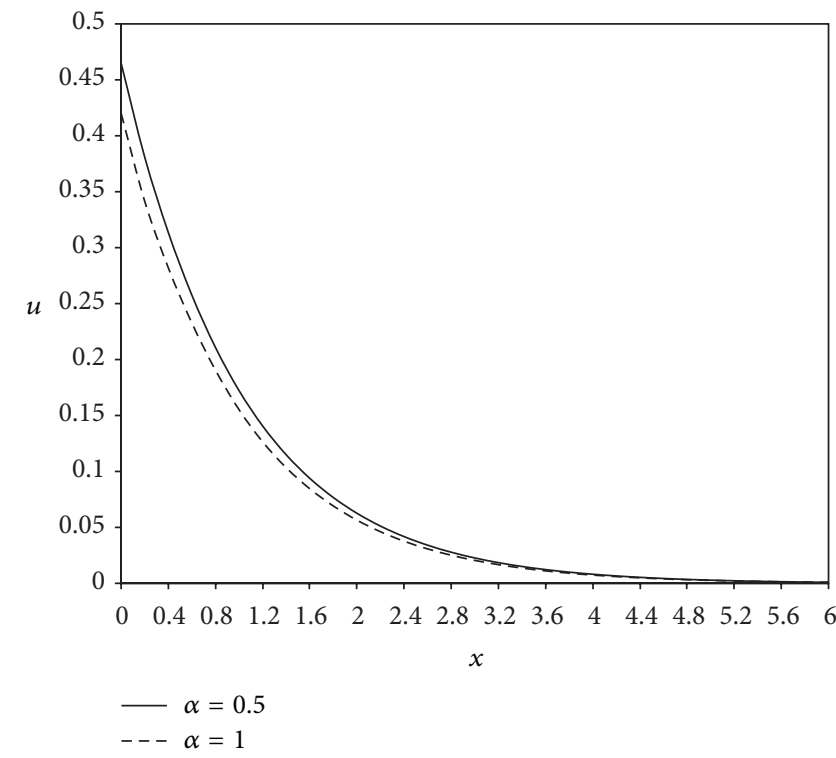

FIGURE 1: Displacement distribution $u$ for different values of $\alpha$ at $t=0.1$.

which depict the variations of displacement, temperature, and stress component. The material chosen for the purpose of numerical evaluations is copper, for which we take the following values of the different physical constants

$E=36.9 \times 10^{10} \mathrm{kgm}^{-1} \mathrm{~s}^{-2}, \varepsilon_{0}=\left(10^{-9} / 36 \pi\right) \mathrm{Fm}^{-1}, k=$ $386 \mathrm{Wm}^{-1} \mathrm{~K}^{-1}, T_{0}=293 \mathrm{~K}, \rho=8954 \mathrm{kgm}^{-3}, \tau_{0}=0.02 \mathrm{~s}$, $v=0.33, \alpha_{t}=1.78 \times 10^{-5} \mathrm{~K}^{-1}, c_{E}=383.1 \mathrm{Jkg}^{-1} \mathrm{~K}^{-1}, H_{0}=$ $\left(10^{7} / 4 \pi\right) \mathrm{Am}^{-1}, \mu_{0}=4 \pi 10^{-7} \mathrm{Hm}^{-1}$.

The general Lame constants $\bar{\lambda}$ and $\bar{\mu}$ are given as

$$
\bar{\lambda}=\frac{E v}{\eta(1+v)(1-2 v)}, \quad \bar{\mu}=\frac{E}{2 \eta(1+v)},
$$

where $\eta$ is the initial stress parameter, $E$ is Young's modulus; and $v$ is Poisson ratio. For isotropic elastic medium with no initial stress, we take $\eta=1$.

The computations are carried out for $t=0.1, t_{0}=0.5$, $\alpha=0.5$, and $\theta^{*}=1$. The numerical technique, outlined in previous section, was used to invert the Laplace transform in (53), providing the displacement, temperature, and stress distributions in the physical domain. The results are represented graphically for different positions of $x$.

Figures 1, 2, and 3 exhibit the space variations of the field quantities in the context of fractional order theory of thermoelasticity with magnetic field and initial stress for different values of fractional parameter $\alpha$.

Figure 1 displays the variations of displacement component $u$ for different values of $\alpha$, and it is noticed that the magnitude of displacement component $u$ decreases with the increase in the value of fractional parameter $\alpha$. In both the cases (i.e., $\alpha=0.5$ and $\alpha=1.0$ ), the displacement component attains maximum value at the boundary of half-space, and then continuously decreases to zero. Hence, displacement component $u$ has similar trend for both the values of $\alpha$.

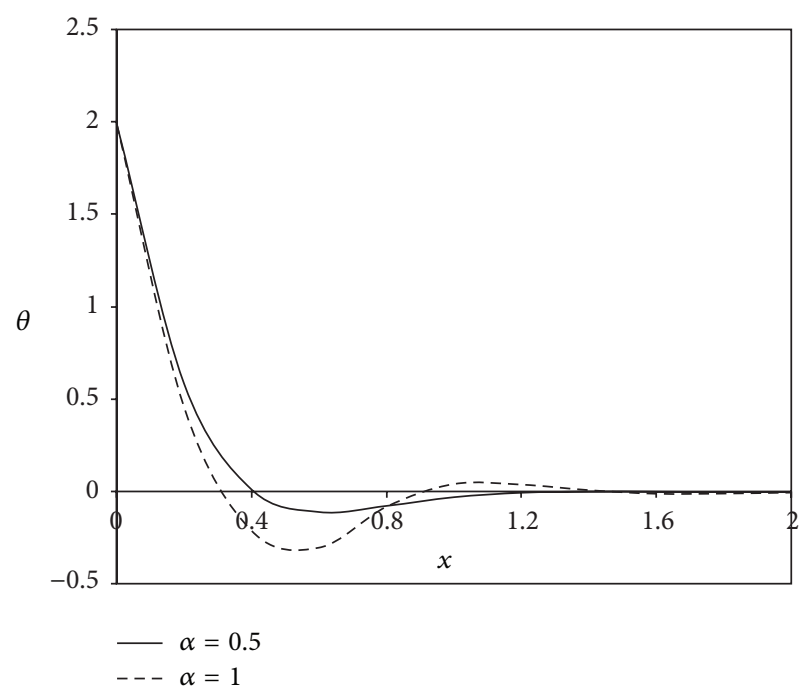

FIgURE 2: Temperature distribution $\theta$ for different values of $\alpha$ at $t=$ 0.1 .



FIGURE 3: Stress distribution $\sigma$ for different values of $\alpha$ at $t=0.1$.

Figure 2 depicts the variations of temperature $\theta$ with distance $x$ for different values of $\alpha$, and it is noticed that in both the cases (i.e., $\alpha=0.5$ and $\alpha=1.0$ ), maximum value of $\theta$ is 2 which is on the boundary of half-space. We observe from the figure that the difference is negligible in the beginning, and with the increase in $x$, the difference is much pronounced up to $x \leq 1.4$; both the series approach to zero. The trends of both the series are alike only up to $x=0.8$.

Figure 3 shows the variations of stress component $\sigma$ with distance $x$ for different values of $\alpha$. It is evident from the figure that both the series have similar trend, that is, first increases to a maximum value and then decreases to a minimum value. The value of $\sigma$ increases with the increase of fractional parameter when $0 \leq x \leq 0.8$, while the trend of change is 


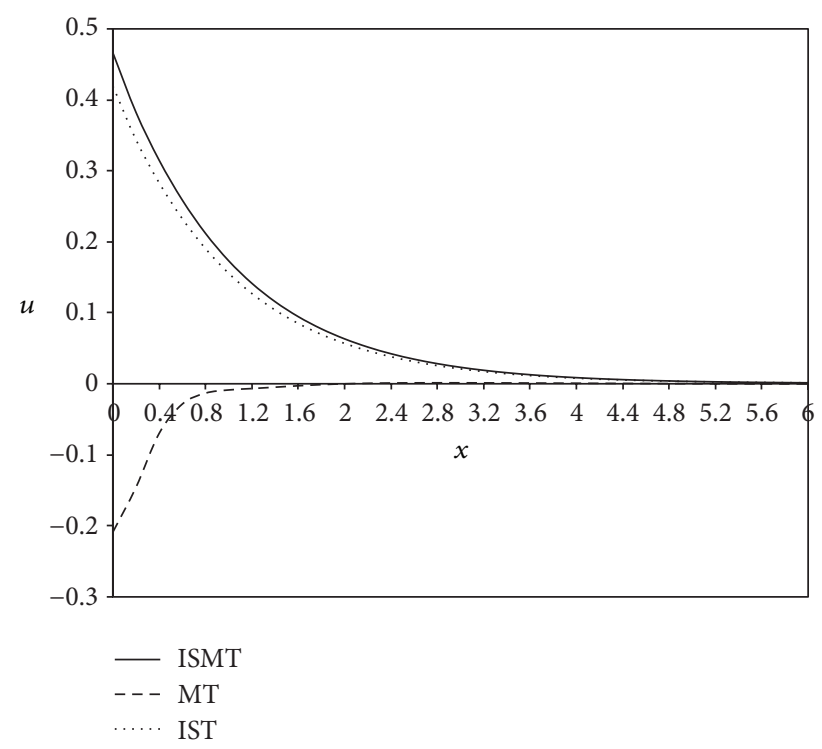

FIgURE 4: Displacement distribution $u$ at $\alpha=0.5, t=0.1$.

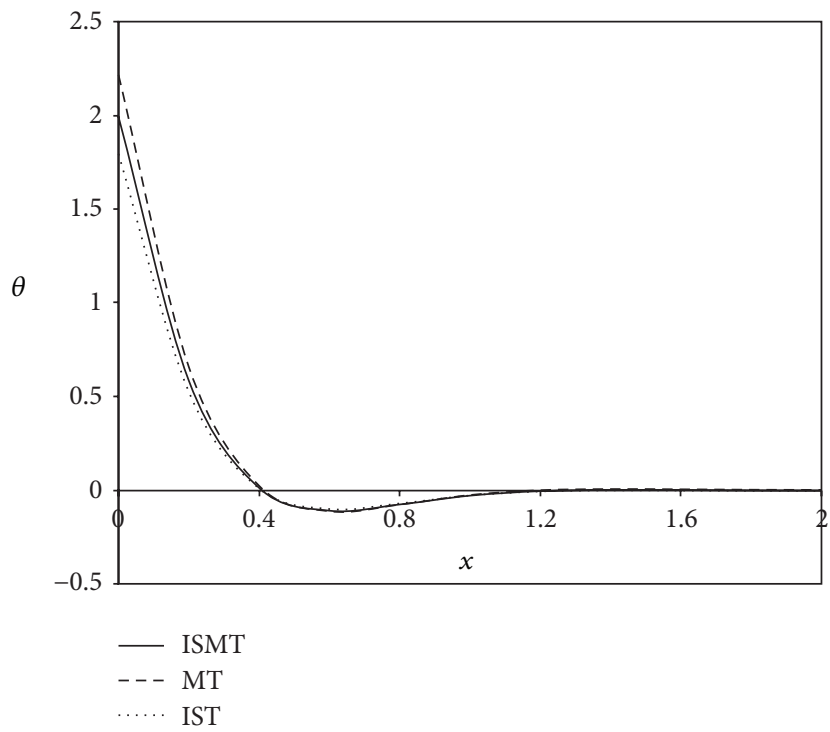

FIGURE 5: Temperature distribution $\theta$ at $\alpha=0.5, t=0.1$.

adverse when $0.8 \leq x \leq 6$. The difference is significant in the range $0 \leq x \leq 2.8$.

Figures 4, 5, and 6 depict the variations of displacement component, temperature, and stress component for three different cases defined as (a) ISMT (initially stressed magnetothermoelasticity), $\eta=2.5, p=1$; (b) MT (magnetothermoelasticity), $\eta=1, p=0$; and (c) IST (initially stressed thermoelasticity), $\eta=2.5, p=1, H_{0}=0$. In all the above cases (a), (b), and (c), the value of fractional order parameter is taken to be $\alpha=0.5$.

Figure 4 shows the variations of displacement component under the three cases discussed in (a), (b), and (c). It is observed that the displacement component in case of ISMT and IST has maximum positive values but in case of MT,

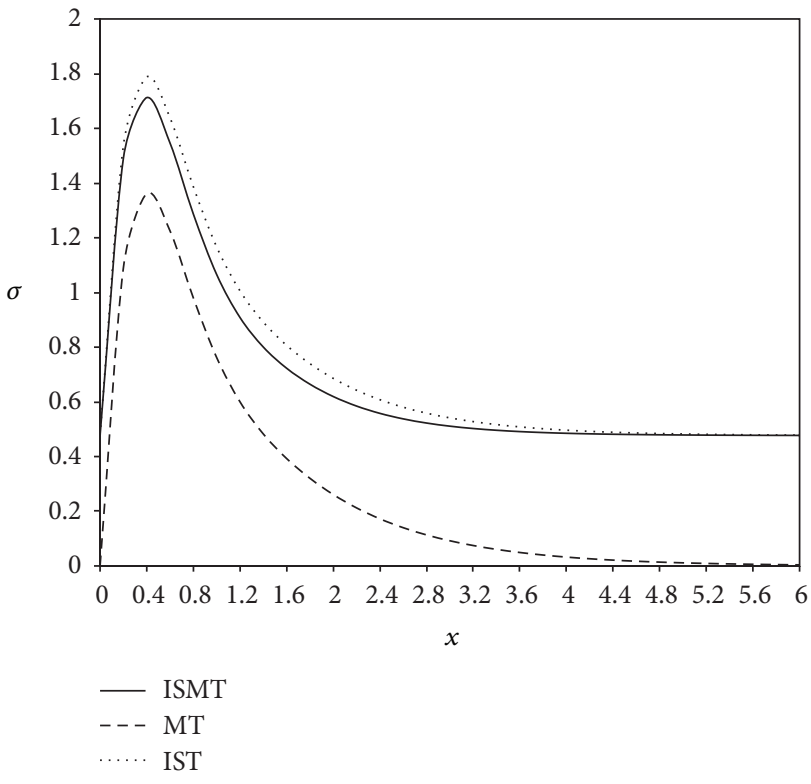

FIGURE 6: Stress distribution $\sigma$ at $\alpha=0.5, t=0.1$.

the displacement component has maximum negative value on the boundary of half-space. The trend for displacement component in case of ISMT and IST is same, that is, both the series are continuously decreasing to zero, but in case of MT, the series rapidly approaches to zero. Figure 4 also exhibits that in the absence of magnetic field (IST) and initial stress (MT) decreases the value of displacement. It is also observed that as compared to ISMT, the difference is significant in case of IST but much pronounced in case of MT.

Figure 5 displays the variations of temperature under the ISMT, MT, and IST theories. It is observed that the trend of the series in case of ISMT, MT, and IST is similar, and the difference is significant. It is found that the absence of initial stress (MT) increases the value of temperature component, but the absence of magnetic field (IST) decreases the value of temperature as compared to the general case (ISMT).

Figure 6 exhibits the variations of stress under ISMT, MT, and IST theories. We found that the behavior of stress component in all the three cases is alike. It also observed that as compared to ISMT theory the stress component has large values in IST theory, but has small values in MT theory. The difference is significant in both IST and MT theories compared with ISMT theory, but the difference is much pronounced in MT theory as compared to IST theory.

Figures 7, 8, and 9 exhibit the variations of displacement component, temperature, and stress component in the context of the fractional order theory of thermoelasticity with magnetic field and initial stress for different values of ramp parameter. The values of ramp parameter are taken as $0.1,0.3$, and 0.5 , and the value of fractional order parameter $\alpha$ is taken to be 0.5 for all the three cases.

Figure 7 demonstrates that as we increase the value of ramp parameter, then the value of displacement component also increases. It is also observed that trend for all the cases is 


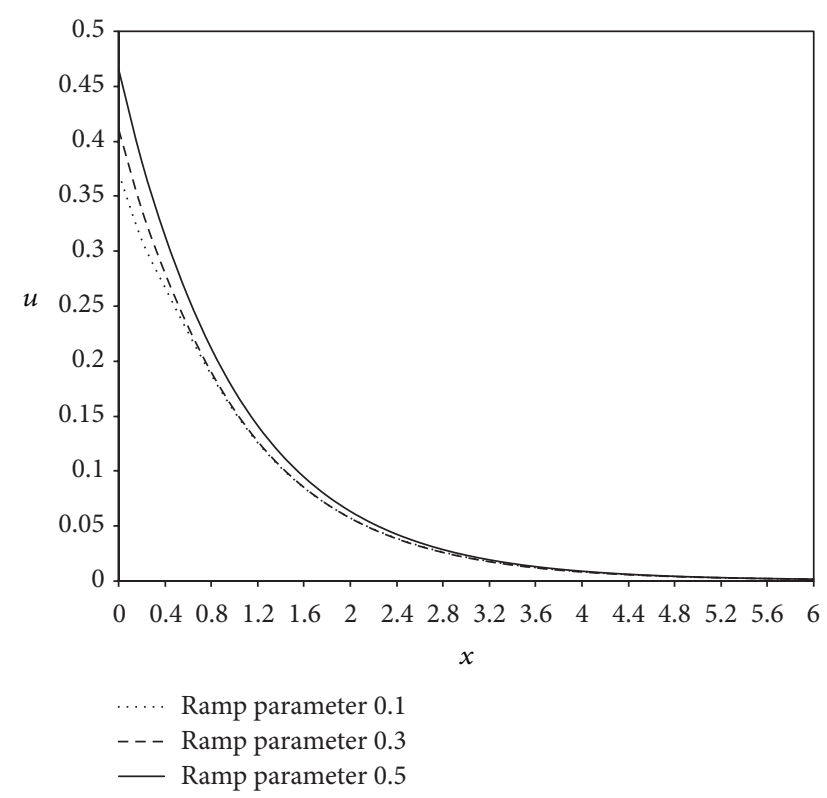

Figure 7: Displacement distribution $u$ for different values of ramp parameter at $\alpha=0.5, t=0.1$.



Figure 8: Temperature distribution $\theta$ for different values of ramp parameter at $\alpha=0.5, t=0.1$.

similar and effect of change in ramp parameter is significant in first two cases and much pronounced in last two cases.

Figure 8 depicts that as we increase the value of ramp parameter, then the value of the temperature rapidly decreases. It is also observed that trend for the series in all the three cases is similar and the effect of change in ramp parameter is much pronounced in the initial range of distance $0 \leq x \leq 1.0$.

Figure 9 displays that as we increase the value of ramp parameter, then the value of the stress component decreases.



FIGURE 9: Stress distribution $\sigma$ for different values of ramp parameter at $\alpha=0.5, t=0.1$.

It is also observed that trend for the series in all the three cases is similar (i.e., all the series have same initial value, and first increases to a maximum value then decreases to a minimum value) and the effect of change in ramp parameter is much pronounced. It is also apparent from the figure that as we increase the value of ramp parameter from 0.1 to 0.3 , the values of stress component decrease rapidly as compared to increase in the value of ramp parameter from 0.3 to 0.5 .

\section{Summary}

We consider a perfectly conducting elastic homogeneous half-space in the context of fractional order generalized thermoelasticity theory with magnetic field and initial stress. The method of the matrix exponential, which constitutes the basis of the state space approach of modern theory, is applied to the nondimensional equations. The importance of state space approach is recognized in the fields, where the time behaviour of physical process is of interest. The state space approach is more general than the classical Laplace and Fourier transform technique. Consequently, state space is applicable to all systems that can be analyzed by integral transform in time and also is applicable to many systems for which transform theory breaks down [41]. The potential function approach is often used to solve problems of thermoelasticity theory. This, however, has several disadvantages as outlined in [39]. These may be summarized in the fact that the boundary conditions for physical problems are related directly to the physical quantities under consideration not to the potential functions. Secondly, more stringent assumptions must be made on the behaviour of potential functions than on the actual physical quantities. Last of all, it was found that many integral representations of physical quantities are convergent in the classical sense, while their potential function representations 
only converge in the mean. All these reasons have led many authors to avoid the use of potential functions. Among the alternatives is the state space formulation. This approach enables one to use the methodology of modern control theory in solving problems of thermoelasticity.

The main conclusions due to the influence of magnetic field, initial stress, fractional parameter, and ramp parameter can be summarized as follows:

(1) the phenomenon of finite speed of propagation is preserved for all the field variables except for stress component due to the presence of initial stress, and all results are in agreement with the generalized theory of thermoelasticity;

(2) the fractional parameter has a significant effect on all the studied fields. The displacement component decreases with the increase in the value of the fractional parameter;

(3) the thermodynamic temperature first decreases then increases, but the stress component first increases then decreases to a minimum value with the increase in the value of fractional parameter;

(4) the effect of magnetic field is silent as compared to initial stress on studied fields. Also, all the field variables for the cases ISMT, MT, and IST behave alike except the displacement component for MT;

(5) in the context of fractional order generalized thermoelasticity theory with magnetic field and initial stress, the increase in the value of ramp parameter increases the magnitude of displacement component but decreases the magnitude of temperature distribution and stress component;

(6) displacement component $u$, temperature distribution $\theta$, and stress component $\sigma$ show almost similar pattern for different values of fractional parameter $\alpha$ and ramp parameter $t_{0}$.

\section{Acknowledgment}

One of the authors, Sandeep Singh Sheoran, is thankful to U. G. C., New Delhi, for the financial support Vide Letter no. F. 17-11/2008 (SA-1).

\section{References}

[1] M. A. Biot, "Thermoelasticity and irreversible thermodynamics," Journal of Applied Physics, vol. 27, pp. 240-253, 1956.

[2] H. W. Lord and Y. Shulman, "A generalized dynamical theory of thermoelasticity," Journal of the Mechanics and Physics of Solids, vol. 15, no. 5, pp. 299-309, 1967.

[3] A. E. Green and K. A. Lindsay, “Thermoelasticity," Journal of Elasticity, vol. 2, no. 1, pp. 1-7, 1972.

[4] R. S. Dhaliwal and H. H. Sherief, "Generalized thermoelasticity for anisotropic media," Quarterly of Applied Mathematics, vol. 38, no. 1, pp. 1-8, 1980.

[5] R. B. Hetnarski and J. Ignaczak, "Generalized thermoelasticity," Journal of Thermal Stresses, vol. 22, no. 4-5, pp. 451-476, 1999.
[6] H. M. Youssef, "Two-dimensional generalized thermoelasticity problem for a half-space subjected to ramp-type heating," European Journal of Mechanics A/Solids, vol. 25, no. 5, pp. 745763, 2006.

[7] L. Knopoff, "The interaction between elastic wave motion and a magnetic field in electrical conductors," Journal of Geophysical Research, vol. 60, pp. 441-456, 1955.

[8] P. Chadwick, "Elastic wave propagation in a magnetic field," in Proceedings of the International Congress of Applied Mechanics, pp. 143-153, Brussels, Belgium, 1957.

[9] S. Kaliski and J. Petykiewicz, "Equation of motion coupled with the field of temperature in a magnetic field involving mechanical and electrical relaxation for anisotropic bodies," Proceedings of Vibration Problems, vol. 4, pp. 1-12, 1959.

[10] G. Paria, "On magneto-thermo-elastic plane waves," Proceedings of the Cambridge Philosophical Society, vol. 58, pp. 527-531, 1962.

[11] A. H. Nayfeh and S. Nemat-Nasser, "Electromagneto-thermoelastic plane waves in solids with thermal relaxation," Journal of Applied Mechanics, Transactions ASME, vol. 39, no. 1, pp. 108113, 1972.

[12] H. H. Sherief and M. A. Ezzat, "A thermal-shock problem in magneto-thermoelasticity with thermal relaxation," International Journal of Solids and Structures, vol. 33, no. 30, pp. 44494459, 1996.

[13] H. H. Sherief and K. A. Helmy, "A two-dimensional problem for a half-space in magneto-thermoelasticity with thermal relaxation," International Journal of Engineering Science, vol. 40, no. 5, pp. 587-604, 2002.

[14] M. A. Ezzat and H. M. Youssef, "Generalized magneto-thermoelasticity in a perfectly conducting medium," International Journal of Solids and Structures, vol. 42, no. 24-25, pp. 63196334, 2005.

[15] A. Baksi, R. K. Bera, and L. Debnath, "A study of magnetothermoelastic problems with thermal relaxation and heat sources in a three-dimensional infinite rotating elastic medium," International Journal of Engineering Science, vol. 43, no. 19-20, pp. 1419-1434, 2005.

[16] M. A. Biot, Mechanics of Incremental Deformation, John Wiley \& Sons, New York, NY, USA, 1965.

[17] A. Chattopadhyay, S. Bose, and M. Chakraborty, "Reflection of elastic waves under initial stress at a free surface: P and SV motion," Journal of the Acoustical Society of America, vol. 72, no. 1, pp. 255-263, 1982.

[18] A. Montanaro, "On singular surfaces in isotropic linear thermoelasticity with initial stress," Journal of the Acoustical Society of America, vol. 106, no. 3, pp. 1586-1588, 1999.

[19] M. I. A. Othman and Y. Song, "Reflection of plane waves from an elastic solid half-space under hydrostatic initial stress without energy dissipation," International Journal of Solids and Structures, vol. 44, no. 17, pp. 5651-5664, 2007.

[20] B. Singh, "Effect of hydrostatic initial stresses on waves in a thermoelastic solid half-space," Applied Mathematics and Computation, vol. 198, no. 2, pp. 494-505, 2008.

[21] M. Caputo and F. Mainardi, "A new dissipation model based on memory mechanism," Pure and Applied Geophysics, vol. 91, no. 1, pp. 134-147, 1971.

[22] M. Caputo and F. Mainardi, "Linear models of dissipation in anelastic solids," La Rivista del Nuovo Cimento, vol. 1, no. 2, pp. 161-198, 1971. 
[23] M. Caputo, "Vibrations of an infinite viscoelastic layer with a dissipative memory," Journal of the Acoustical Society of America, vol. 56, no. 3, pp. 897-904, 1974.

[24] R. Gorenflo and F. Mainardi, "Fractional calculus: integral and differential equations of fractional orders," in Fractals and Fractional Calculus in Continuum Mechanics, vol. 378, pp. 223276, Springer, Vienna, Austria, 1997.

[25] R. Hilfer, Application of Fraction Calculus in Physics, World Scientific Publishing, Singapore, 2000.

[26] J. Ignaczak and M. Ostoja-Starzewski, Thermoelasticity with Finite Wave Speeds, Oxford University Press, Oxford, UK, 2010.

[27] L. Debnath and D. Bhatta, Integral Transforms and Their Applications, Chapman \& Hall/CRC, Taylor \& Francis Group, London, UK, 2nd edition, 2007.

[28] I. Podlubny, Fractional Differential Equations, vol. 198, Academic Press, New York, NY, USA, 1999.

[29] Y.Z. Povstenko, "Fractional heat conduction equation and associated thermal stress," Journal of Thermal Stresses, vol. 28, no. 1, pp. 83-102, 2005.

[30] H. H. Sherief, A. M. A. El-Sayed, and A. M. Abd El-Latief, "Fractional order theory of thermoelasticity," International Journal of Solids and Structures, vol. 47, no. 2, pp. 269-275, 2010.

[31] H. M. Youssef, "Theory of fractional order generalized thermoelasticity," Journal of Heat Transfer, vol. 132, no. 6, pp. 1-7, 2010.

[32] M. A. Ezzat, "Thermoelectric MHD non-Newtonian fluid with fractional derivative heat transfer," Physica B, vol. 405, no. 19, pp. 4188-4194, 2010.

[33] M. A. Ezzat, "Magneto-thermoelasticity with thermoelectric properties and fractional derivative heat transfer," Physica B, vol. 406, no. 1, pp. 30-35, 2011.

[34] G. Jumarie, "Derivation and solutions of some fractional BlackScholes equations in coarse-grained space and time. Application to Merton's optimal portfolio," Computers \& Mathematics with Applications, vol. 59, no. 3, pp. 1142-1164, 2010.

[35] A. S. El-Karamany and M. A. Ezzat, "Convolutional variational principle, reciprocal and uniqueness theorems in linear fractional two-temperature thermoelasticity," Journal of Thermal Stresses, vol. 34, no. 3, pp. 264-284, 2011.

[36] A. S. El-Karamany and M. A. Ezzat, "On fractional thermoelasticity," Mathematics and Mechanics of Solids, vol. 16, no. 3, pp. 334-346, 2011.

[37] A. S. El-Karamany and M. A. Ezzat, "Fractional order theory of a perfect conducting thermoelastic medium," Canadian Journal of Physics, vol. 89, no. 3, pp. 311-318, 2011.

[38] S. Kothari and S. Mukhopadhyay, "A problem on elastic half space under fractional order theory of thermoelasticity," Journal of Thermal Stresses, vol. 34, no. 7, pp. 724-739, 2011.

[39] L. Y. Bahar and R. B. Hetnarski, "State space approach to thermoelasticity," Journal of Thermal Stresses, vol. 1, pp. 135-147, 1978.

[40] G. Honig and U. Hirdes, "A method for the numerical inversion of Laplace transforms," Journal of Computational and Applied Mathematics, vol. 10, no. 1, pp. 113-132, 1984.

[41] D. Wiberg, Theory and Problems of State Space and Linear Systems. Schaum's Outline Series in Engineering, Mc Graw-Hill, New York, NY, USA, 1971. 


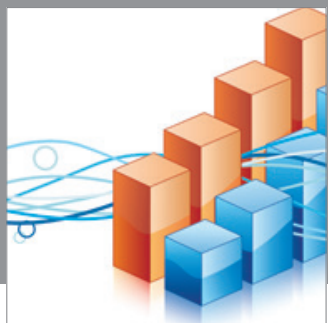

Advances in

Operations Research

mansans

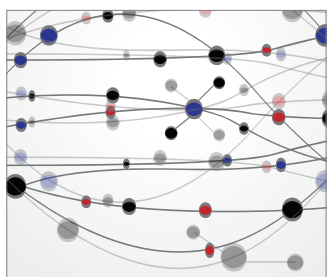

The Scientific World Journal
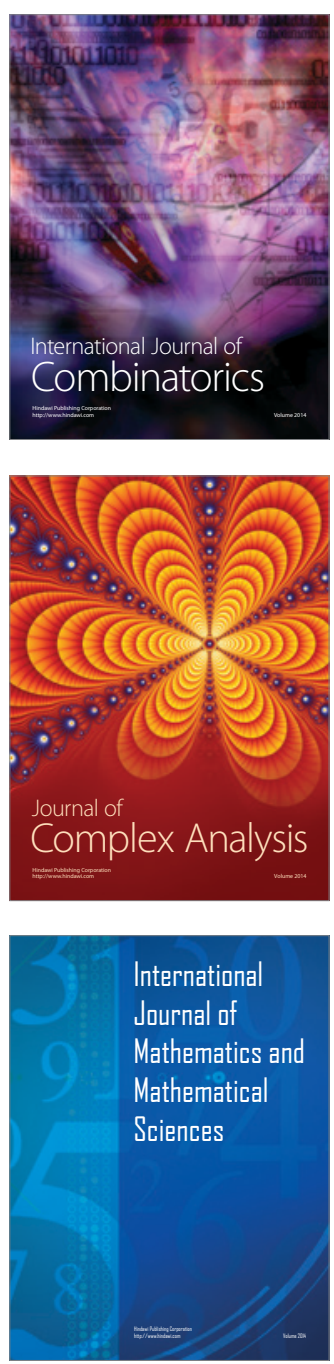
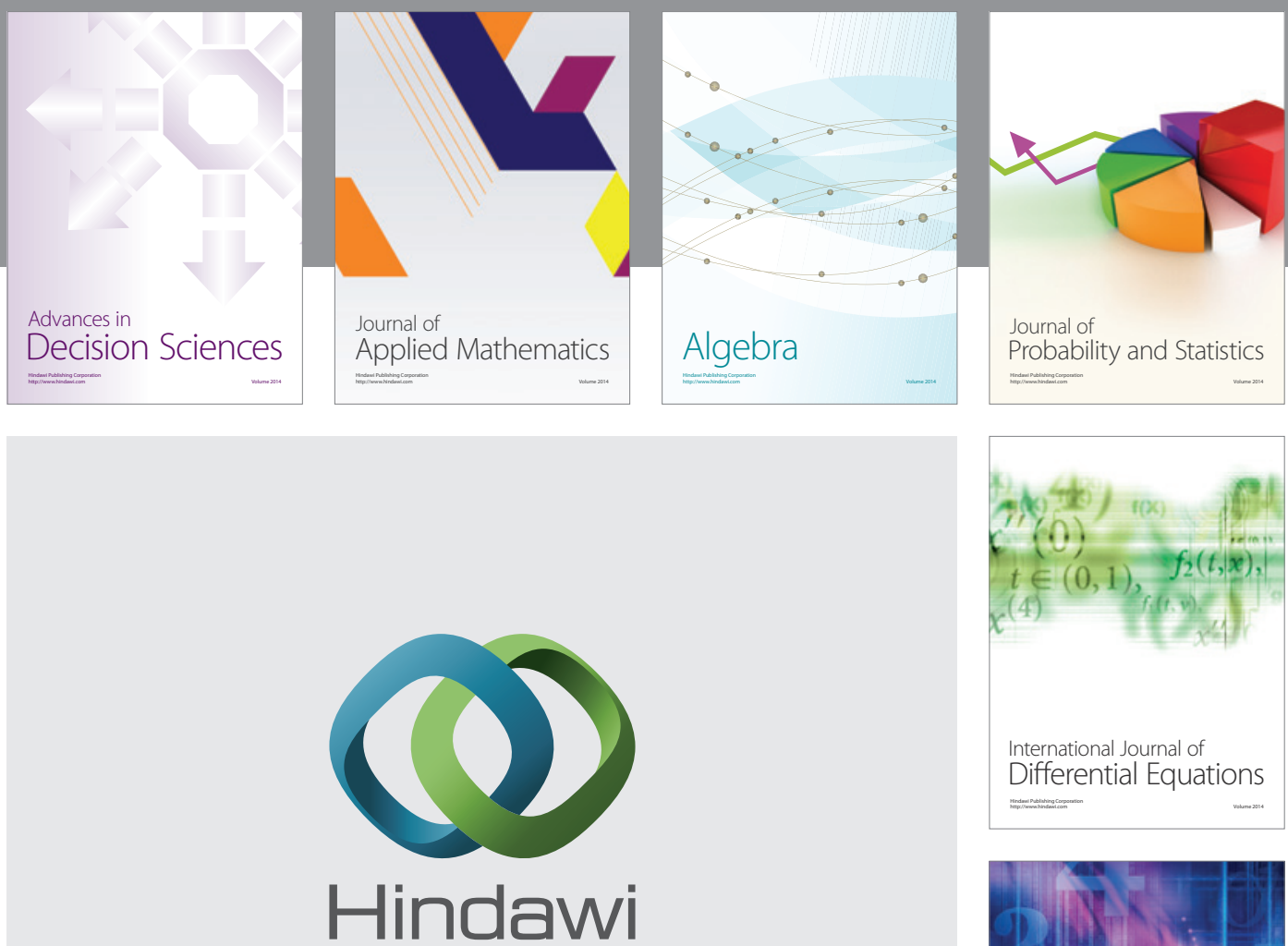

Submit your manuscripts at http://www.hindawi.com
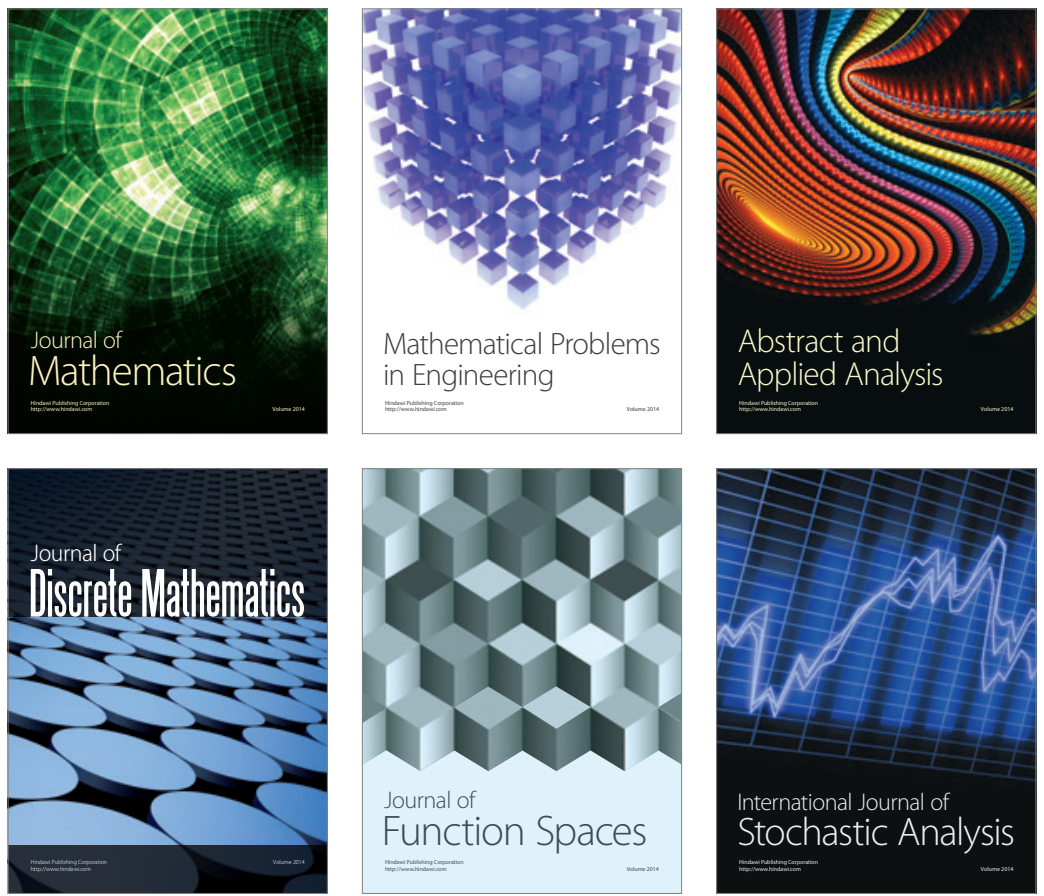

Journal of

Function Spaces

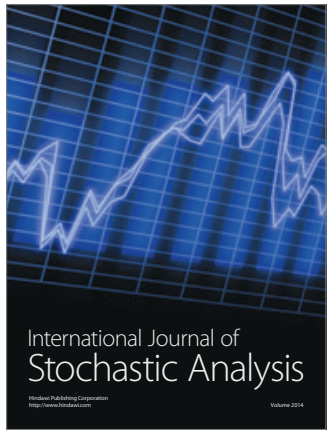

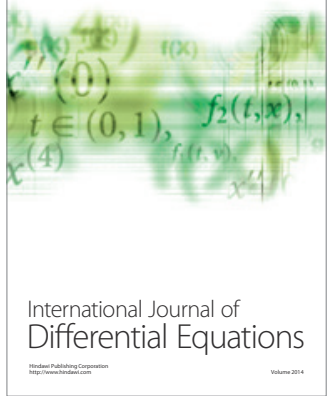
